\title{
On a Self.Exciting Process in Magneto-Hydrodynamics (II).
}

\author{
By \\ Hitoshi Takeuchi and Yasuo Shimazu. \\ Geophysical Institute, Faculty of Science, Tokyo University, Tokyo.
}

\begin{abstract}
The present paper is concerned with the problem of the self-exciting process in magneto-hydrodynamics studied in a previous paper (referred to as Paper I). In $\$ 2$ and $\S 3$, by solving a differential equation numerically, the results in Paper I are checked in a conclusive way. The existence of a self-exciting dynamo having thus been shown, the detailed structure of this dynamo is studied in $\$ 4$ and $\$ 5$. In $\$ 6$, a new approximate method of solving the problem is proposed and applied to the problem in Paper I. This method of approximation is found to be fairly useful. By using this method, it is shown in $\$ 7$ that the self-exciting dynamo is possible by the fluid motions of considerable varieties. The orders of magnitudes of the fluid velocities required are found to be the same as that in Paper I.
\end{abstract}

$\$ 1$. In a previous paper (which will be referred to hereafter as Paper I (1952)), the present writers showed that a self-exciting dynamo is possible by which the earth's main magnetic field may be produced and maintained. The self-exciting dynamo considered was supposed to have the structure similar to that supposed by W.M. Elssasser (1946, 1947) and E.C. Bullard $(1948,1949)$ in their studies of the secular variations of the earth's magnetic field. In the present paper, using the results obtained in Paper I, further studies will be made on this self-exciting dynamo.

$\$ 2$. In our self-exciting dynamo, the velocity field (relative to the earth's mantle) of the conducting fluid in the earth's core is assumed to be represented by arbitrary linear combinations of the following two velocity fields. $T_{1}{ }^{0}-$ type velocity field

$$
\begin{aligned}
& \left\{\begin{array}{l}
0, \\
0, \\
V_{1}{ }^{\prime}(r) r \\
P_{1} P_{1},
\end{array}\right. \\
& P_{1}=\cos \theta, \\
& V_{1}{ }^{\prime \prime}(r)=\frac{1}{a} V_{1,0}\left[1-\left(\begin{array}{l}
r \\
a
\end{array}\right)\right],
\end{aligned}
$$

$S_{2}{ }^{2 c \ldots}$ type ve'ocity field

$$
\left\{\begin{array}{l}
-6 r V_{2}^{2 c}(r) \cos 2 \phi P_{2}^{2}, \\
-\left(r^{2} \frac{d V_{2}^{2 c}}{d r}+3 r V_{2}^{2 c}\right) \frac{\partial\left(\cos 2 \phi P_{2}^{2}\right)}{\partial \theta} \\
-(\quad " \quad) \frac{\partial(" \prime)}{\sin \theta \partial \phi},
\end{array}\right.
$$

$$
\begin{aligned}
& P_{2}{ }^{2}=3 \sin ^{2} \theta \\
& V_{2}{ }^{2} c(r)=\frac{1}{a} V_{2,2 c}\left[1-\left(\begin{array}{l}
r \\
a
\end{array}\right)\right]^{2} .
\end{aligned}
$$$$
\text { ................... (2.1) }
$$

In $(2.1)$, the $(r, \theta, \phi)$ components of the velocities refer to the polar axis which coincides with the rotation axis of the model earth, and $a$ is the radius of the earth's core. $V_{1,0}$ and $V_{2,2 c}$ are certain constants having the dimensions of velocity. Velocities given in (2.1) become zero at $r=a$. As was pointed out by Bullard (1949), the $T_{1}^{\prime \prime}$-type velocity in the earth's core is considered to be much more predominant than the $S_{.2}{ }^{2}$-type velocity. So we shall, hereafter, confine ourselves to the study of the case in which $V_{1,0} \rightarrow \infty$.

In order to study this case, we must solve the following eigen-value problem. (see $\$ 5$, Paper I).

$$
\begin{aligned}
& \frac{d^{4} \bar{T}_{2}^{0}}{d \xi^{0}}+\frac{12}{\xi} d^{3} \bar{T}_{2}^{0} d \xi^{0}+\frac{28 d^{2} \bar{T}_{2}^{0}}{\xi^{2}} d \xi^{2}+{ }_{35}^{36} \times 12 y^{2}(1-\xi)^{*} \xi \\
& \quad \times\left[\xi(1-\xi) \frac{d \bar{T}_{2}{ }^{0}}{d \xi}+(7-11 \xi) \bar{T}_{2}{ }^{0}(\xi)\right]=0, \quad(2.2)
\end{aligned}
$$

under the boundary conditions $\bar{T}_{2}{ }^{0}(\xi)=0, \ldots \ldots \ldots \ldots \ldots \ldots \ldots \ldots \ldots \ldots \ldots \ldots$

and

$$
Q(\xi)=\frac{d^{3} \bar{T}_{2}^{0}}{d \xi^{3}}+10 \frac{d^{2} \bar{T}_{2}^{0}}{d \xi^{2}}+18^{d T_{1}^{0}} \frac{T^{0}}{d \xi}=0
$$


at $\xi=1 . \quad$ In $(2.2)$

$$
\xi=\frac{r}{a} \text { and } y=4 \pi \kappa a V_{2,2 c},
$$

$\kappa$ being the conductivity of the earth's core. In $\$ 5$ in Paper $I$, the eigen-value $Y=\frac{8}{3} y$ was found to be at about $Y=100$. In order to obtain the exact value for $Y$, the determinant $\Delta$ in (5.11) in Paper $I$ is evaluated for several $Y$ 's. The results obtained are shown in Table I and in Fig. 1. The exact eigen-value is thus shown to be at $Y=130.6\left(y^{2}=2400\right)$.

Table I. Values of $\Delta$ for $Y$ 's.

\begin{tabular}{r|c|c}
\hline$y^{2}$ & $Y$ & $\Delta$ \\
\hline 0 & 0 & +18 \\
1200 & 92.37 & +12 \\
1658 & 108.5 & +8.2 \\
2400 & 130.6 & -0.3 \\
3600 & 160.0 & -23 \\
4800 & 184.8 & -63 \\
\hline
\end{tabular}

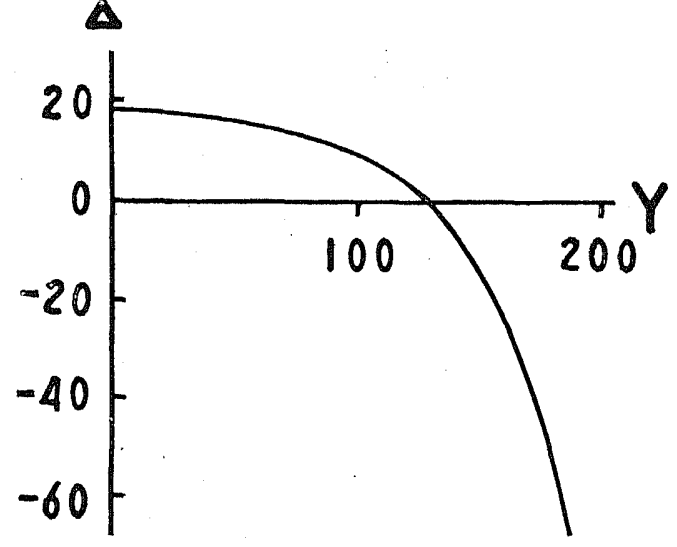

Fig. 1. $Y-\Delta$ Curve.

\$3. In Table II $\left(y^{\prime \prime}=2400\right)$ and Table III $\left(y^{*}=0\right.$ and 4800), are shown the solutions of (2.2) for which $Q(\xi)$ in (2.4) becomes zero at $\xi=1$. These solutions are obtained by making adequate linear combinations of the solutions I and II (defined by (5.8) in Paper I) for each $y^{2}$, and satisfy one of the two imposed boundary conditions.

Table II. Solution for $y^{2}=2400$.

\begin{tabular}{|c|c|c|c|c|c|}
\hline$\xi$ & $\bar{T}_{y^{0}}$ & $\bar{T}_{2}{ }^{\prime \prime}$ & $\bar{T}_{2}{ }^{0 \prime \prime}$ & $\bar{T}_{2}^{0 \prime \prime \prime \prime}$ & $\bar{T}_{2}^{(1) \prime \prime \prime \prime \prime}$ \\
\hline $\begin{array}{l}0 \\
0.1 \\
0.2 \\
0.3 \\
0.4 \\
0.5 \\
0.6 \\
0.7 \\
0.8 \\
0.9 \\
1.0\end{array}$ & $\begin{array}{l}1 . \\
0.8706906 \\
0.7167810 \\
0.4687736 \\
0.07013331 \\
-0.4210779 \\
-0.8152594 \\
-0.9294623 \\
-0.7436155 \\
-0.8851389 \\
0.00522213\end{array}$ & $\begin{array}{r}-1.281836 \\
-1.334939 \\
-1.870458 \\
-3.204420 \\
-4.677167 \\
-4.803987 \\
-2.742478 \\
0.487666 \\
2.986053 \\
3.919917 \\
3.801665\end{array}$ & $\begin{array}{c}0 \\
-1.940291 \\
-9.452420 \\
-16.11707 \\
-10.57754 \\
9.721807 \\
29.60671 \\
31.41339 \\
17.13300 \\
2.659509 \\
-3.761189\end{array}$ & $\begin{array}{c}0 \\
-49.48336 \\
-88.86093 \\
-22.7512 \\
139.9516 \\
237.7424 \\
125.7603 \\
-84.74552 \\
-169.7589 \\
-105.6746 \\
-30.81803\end{array}$ & $\begin{array}{c}0 \\
-667.0607 \\
37.495 .14 \\
1301.714 \\
1663.017 \\
-8.73218 \\
-2018.81 \\
-1747.611 \\
76.10816 \\
924.9716 \\
175.1291\end{array}$ \\
\hline & $(0.00562)$ & $(3.805)$ & $(-3.876)$ & $(-26.60)$ & \\
\hline
\end{tabular}

Table III. Solutions for $y^{2}=4800$ and $y^{2}=0$.

\begin{tabular}{|c|c|c|c|c|c|}
\hline$\xi$ & $\bar{T}_{20}$ & $\bar{T} y^{\prime \prime \prime}$ & $\bar{T}_{2}{ }^{\prime \prime \prime \prime}$ & $\bar{T}_{2}{ }^{0 \prime \prime \prime \prime}$ & $T_{2}{ }^{0 \prime \prime \prime \prime \prime \prime}$ \\
\hline $\begin{array}{l}0 \\
0.1 \\
0.2 \\
0.3 \\
0.4 \\
0.5 \\
0.6 \\
0.7 \\
0.8 \\
0.9 \\
1.0\end{array}$ & $\begin{array}{c}1 \\
-0.528148 \\
-2.00586 \\
-2.97298 \\
-2.33719 \\
0.561066 \\
4.29736 \\
6.03172 \\
4.45712 \\
0.749519 \\
-3.410270\end{array}$ & $\begin{array}{c}-15.2793 \\
-15.2677 \\
-13.5976 \\
-3.73610 \\
17.92768 \\
37.5650 \\
31.7832 \\
0.54075 \\
-29.6135 \\
-41.4841 \\
-40.4950\end{array}$ & $\begin{array}{c}0 \\
1.70375 \\
43.9570 \\
162.5292 \\
246.823 \\
99.4471 \\
-216.182 \\
-356.045 \\
-215.848 \\
-35.3183 \\
39.0966\end{array}$ & $\begin{array}{c}0 \\
107.389 \\
828.173 \\
1351.721 \\
-\quad 76.1808 \\
-2781.986 \\
-2834.92 \\
229.517 \\
2067.85 \\
1288.14 \\
337.942\end{array}$ & $\begin{array}{r}0 \\
3893.37 \\
9052.79 \\
-1999.88 \\
-25773.3 \\
-10417.4 \\
-20351.0 \\
31746.4 \\
2618.63 \\
-12791.16 \\
-43761.8\end{array}$ \\
\hline & $(-3.4101)$ & $(-40.417)$ & $(36.230)$ & $(348.73)$ & \\
\hline
\end{tabular}

Solution for $y^{2}=0$ is $\bar{T}_{2}^{0}=1$ for all $\xi$. 
As is seen from Table II, for the exact eigen-value $y^{2}=2400$, the other boundary condition (2.3) is satisfied simultaneously. For the solution given in Table III, this is not the case. $\bar{T}_{2}{ }^{0(n)}$ 's $(n=0,1,2,3,4)$ in Table II and III are connected with each other by the equation (2.2). A rough numerical check for the results in Table II and III is made as follows. Executing, for example, the integration $\int_{0}^{\xi} \bar{T}_{2}{ }^{0 \prime \prime \prime \prime}(\xi) d \xi$ numerically, we have $\bar{T}_{2}{ }^{\prime \prime \prime}(\xi)$ for each $\xi$. The values of $\bar{T}_{2}{ }^{\prime \prime \prime \prime}(\xi)$ thus obtained should agree with those obtained by direct numerical integration of (2.2). The values of $\int_{0}^{1} \bar{T}_{2}{ }^{0 \prime \prime \prime \prime}(\xi) d \xi, \ldots$, $\int_{0}^{1} \bar{T}_{2}^{0 \prime}(\xi) d \xi$ (Simpson's method of numerical integration is used) are shown in the brackets in Tables II and III. These values agree with $\overline{T_{2}{ }^{0 \prime \prime \prime}}(\xi=1), \ldots, \quad T_{2}^{0}(\xi=1)$ satisfactorily well. Small discrepancies among these values are probably due to the roughness of the Simpson's method of numerical integration. In short, in the last and the present sections, we have shown conclusively the existence of the real eigen-value $y^{2}=2400$ ( $Y$ 130.6) for the equation (2.2) with (2.3) and (2.4). A self-exciting dynamo is thus found by which the earth's main magnetic field may be produced and maintained. Having thus got the exact eigen-value, we shall now study the detailed structure of the self-exciting dynamo.

$\$ 4$. In studying the structure of the selfexciting dynamo, uses will be made of the following expressions for the $(r, \theta, \phi)$ components of the velocity and the magnetic fields.

$T_{1}{ }^{0} \cdots$ type velocity field

$$
\begin{aligned}
& V\left\{\begin{array}{l}
0, \\
0, \\
-\frac{V_{1,0}}{4} 4 \xi V_{1}^{0}(\xi) \sin \theta,
\end{array}\right.
\end{aligned}
$$

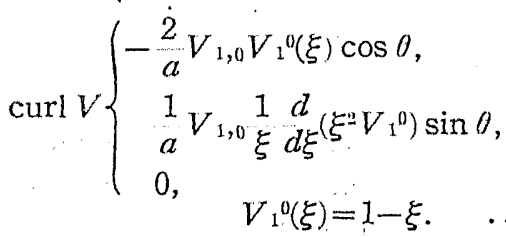

$S_{2}{ }^{2 c}$-type velocity field

$$
V\left\{\begin{array}{l}
-\frac{8}{3} V_{2,2 c} \cdot \frac{27}{4} \xi V_{2}^{2 c}(\xi) \cos 2 \phi \sin ^{2} \theta, \\
-3 V_{2,2 c}\left(\xi^{\frac{d}{d V_{2}^{2 c}}} \frac{{ }^{2 c}}{d \xi}+3 \xi V_{2}^{2 c}\right) \cos 2 \phi \sin 2 \theta, \\
6 V_{2,2 c}(\quad) \sin 2 \phi \sin \theta,
\end{array}\right.
$$

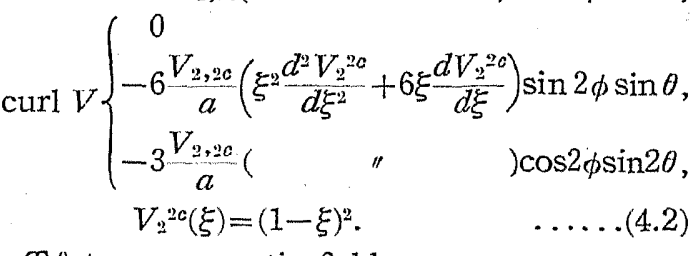

$T_{2}{ }^{0}$-type magnetic field

$$
H\left\{\begin{array}{l}
0 \\
0 \\
-6 \xi^{2} \bar{T}_{2} 0(\xi) X \sin 2 \theta,
\end{array}\right.
$$

$\operatorname{curl} H\left\{\begin{array}{l}-24 \frac{X}{a} \xi \bar{T}_{2} 0(\xi) \cdot \frac{1}{4}(3 \cos 2 \theta+1), \\ 6 \frac{X}{a} \xi\left(\xi \frac{d \bar{T}_{2}{ }^{0}}{d \xi}+3 \bar{T}_{2}^{0}\right) \sin 2 \theta, \\ 0, \\ X=4 \pi \kappa a \cdot \frac{V_{1,0}}{4} .\end{array}\right.$

$\mathrm{S}_{1}{ }^{0}$-type magnetic field

$$
H\left\{\begin{array}{l}
-\frac{2 S_{1}{ }^{0}(\xi)}{0} \cos \theta \\
\left(\xi \frac{d S_{1}^{0}}{d \xi}+2 S_{1}^{0}\right) \sin \theta \\
0
\end{array}\right.
$$

$\operatorname{curl} H\left\{\begin{array}{l}0, \\ 0, \\ \frac{1}{a}\left(\xi^{\frac{d^{2} S_{1}{ }^{0}}{d \xi^{2}}+4}+\frac{d S_{1}{ }^{0}}{d \xi}\right) \sin \theta,\end{array}\right.$

$$
S_{1}{ }^{0}(\xi)=-\frac{3}{2}\left(\xi^{d-\bar{T}_{2}^{0}} d \xi^{2}+6 \frac{d \bar{T}_{2}^{0}}{d \xi}\right) .
$$

$T_{2}^{2 s-t y p e ~ m a g n e t i c ~ f i e l d ~}$

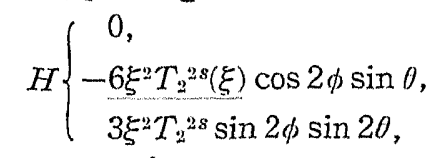

$\operatorname{curl} H\left\{\begin{array}{l}-18 \frac{\xi}{a} T_{2}{ }^{2 s}(\xi) \sin 2 \phi \sin ^{2} \theta \\ -3 \frac{1}{a}\left(\xi^{2} \frac{d T_{2}^{2 s}}{d \xi}+3 \xi T_{2}^{28}\right) \sin 2 \phi \sin 2 \theta, \\ -6 \frac{1}{a}\left({ }^{\prime \prime}\right) \cos 2 \phi \sin \theta\end{array}\right.$

$$
\begin{array}{r}
T_{2}{ }^{28}(\xi)=\frac{3}{7} y\left[\xi(1-\xi) \frac{d \bar{T}_{2}{ }^{0}}{d \xi}+(7-11 \xi) \bar{T}_{2}{ }^{0}\right] \\
y^{2}=2400 .
\end{array}
$$


The values of $4 \xi V_{1}^{0}(\xi)$ and $\frac{27}{4} \xi V_{2}^{20}(\xi)$ (see the equations (4.1) and (4.2)) are shown in Table IV. The values of $6 \xi^{2} \bar{T}_{2}{ }^{0}, 2 S_{1}{ }^{0},\left(\xi S_{1}{ }^{0 \prime}\right.$ $+2 S_{1}{ }^{0}$ ), and $6 \xi^{2} T_{2}{ }^{2 s}$ (see the equations (4.3), (4.4) and (4.5)) are shown in Table. $V$ and in Figs. 2,3 and $4,2 S_{1}{ }^{0}(\xi=1)$ being taken $2 S_{1}{ }^{0}(\xi=1)=1$ tentatively.

Table V. Radial variations of magnetic fields.

Table IV. Radial variations of velocity fields.

\begin{tabular}{l|l|l}
\hline$\xi$ & $4 \xi(1-\xi)$ & $\frac{27}{4} \xi(1-\xi)^{2}$ \\
\hline 0 & 0 & 0 \\
0.1 & 0.36 & 0.54675 \\
0.2 & 0.64 & 0.864 \\
0.3 & 0.84 & 0.99275 \\
0.4 & 0.96 & 0.9720 \\
0.5 & 1.00 & 0.84375 \\
0.6 & 0.96 & 0.6480 \\
0.7 & 0.84 & 0.42525 \\
0.8 & 0.64 & 0.2160 \\
0.9 & 0.36 & 0.06075 \\
1.0 & 0 & 0 \\
\hline
\end{tabular}

\begin{tabular}{c|c|c|c|c}
\hline$\xi$ & $6 \xi^{2 \bar{T}_{2}{ }^{0}}$ & $2 S_{1}{ }^{0}$ & $\xi S_{1}{ }^{1 \prime}+2 S_{1}{ }^{0}$ & $6 \xi^{2} T_{2 s}^{2 s}$ \\
\hline 0 & 0 & 0.403753 & 0.40375 & 0 \\
0.1 & $0.0^{3} 914169$ & 0.430666 & 0.47931 & 0.773998 \\
0.2 & $0.0^{2} 301030$ & 0.688403 & 1.12906 & 1.93851 \\
0.3 & $0.0^{2} 442964$ & 1.263152 & 2.20530 & 1.47394 \\
0.4 & $0.0^{2} 117817$ & 1.695332 & 1.88497 & -2.32076 \\
0.5 & -0.0110526 & 1.257983 & -1.19524 & -7.06826 \\
0.6 & -0.0308149 & -0.068727 & -4.52103 & -5.46677 \\
0.7 & -0.0478179 & -1.307977 & -4.25821 & 5.69262 \\
0.8 & -0.0499679 & -1.660091 & -1.32671 & 17.93345 \\
0.9 & -0.0328391 & -1.360353 & 0.44662 & 18.40224 \\
1.0 & 0 & -1.000000 & 0.50000 & 0 \\
\hline
\end{tabular}

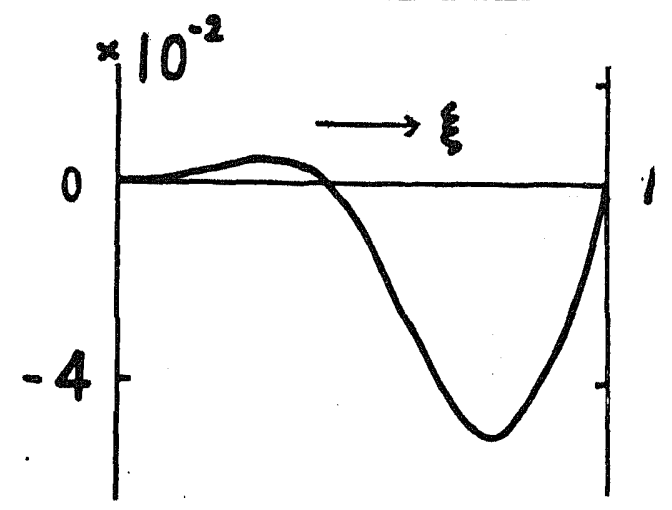

Fig. 2. $\quad 6 \xi^{2} \bar{T}_{2}^{0}$

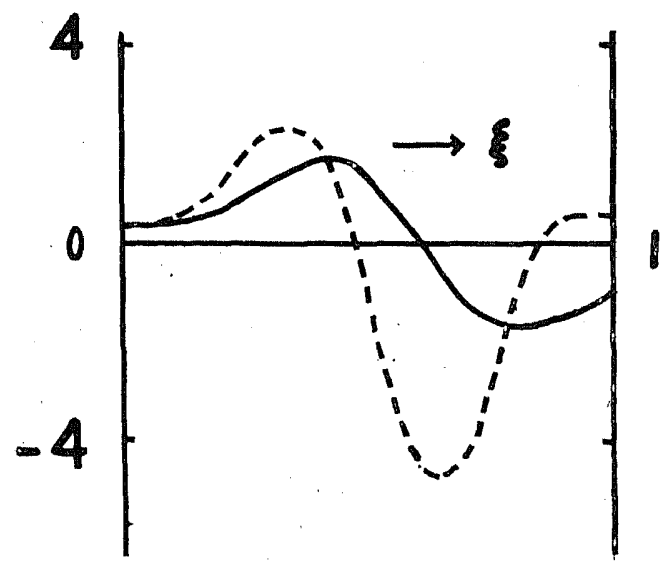

Fig. 3. $-2 S_{1}^{0},---\xi\left(S_{1}^{0}\right)^{\prime}+2 S_{1}^{0}$

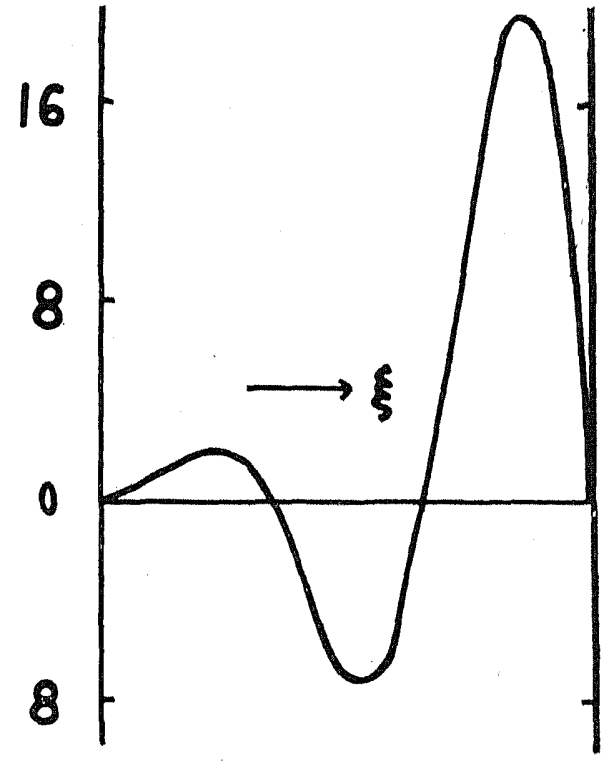

Fig. 4. $6 \xi^{2} T_{2}^{2 s}$

The maximum value of $6 \xi^{2} \bar{T}_{2}^{0}$ (and aiso of $\left.6 \xi^{2} T_{2}{ }^{2 s}\right)$ is at about $\xi=0.8$, and the ratio of $6 X \xi^{2} \bar{T}_{2}{ }^{0}$ (the maximum absolute value throughout the earth's core of the $T_{2}{ }^{0} \ldots$ type magnetic field) to $2 S_{1}^{0}(\xi=1)$ (the maximum value at the surface of the earth's core of the $S_{1}{ }^{0}$ type magnetic field) is about 


$$
\frac{6 X \xi^{2} \bar{T}_{2}{ }^{0}}{2 S_{1}{ }^{0}}=0.05 X
$$

Taking $2 S_{1}{ }^{0}(\xi=1)=3$ gauss and taking $X$ $=1000$ (see Table II in Paper I) - tentatively, we have in the earth's core, the $T_{2}{ }^{0}$-type magnetic field of about 150 gauss. This result is worthy of notice. In his study on the secular variations in the earth's magnetic field, Bullard (1948) concludes that many of the observed facts can be accounted for by an induction current caused by a circular motion with a diameter of a few hundred kilometers situated near the surface of the core, but in order to get numerical agreement, an improbably strong (inducing) field at the core has to be assumed. This difficulty is, however, removed by taking the above self-exciting dynamo into consideration. This dynamo, besides giving us an explanation for the maintenance of the dipole magnetic field of the earth, provides us also a way to have a large magnetic field in the core. The secular variations of the earth's magnetic field may be caused by an eddy motion of rather local type in the presence of this large magnetic field.

$\$ 5$. In this section, we shall examine the case when $X=4 \pi \kappa a \cdot \frac{V_{1,0}}{4}=1000$. Since the value $X=10^{3}$ is taken rather tentatively, great importance should not be attached to the results in this section. Furthermore, the results may be changed drastically by the future hydrodynamical studies on the fluid motion in the earth's core.

[1] In the expression for $X, \frac{V_{1,0}}{4}$ is the maximum value of the $T_{1}{ }^{0}$-type velocity field. Taking $a=3.5 \times 10^{8} \mathrm{~cm}$. and $r=3 \times 10^{-0}$ e.m.u. as are used usually, we have $\frac{V_{1,0}}{4}$ $=0.076 \frac{\mathrm{cm} \text {. }}{\mathrm{sec} .}$ The value of $Y$ for this case is obtained as follows. As is seen from Table II in Paper I, the approximate value of $Y$ for this case is nearly the same as $X=\infty$. In short, $X=10^{3}$ is taken to be the same as $X=\infty$ from the physical point of view. Thus it may be allowed to take $Y=130$ (see Table I) for $X=1000$. The corresponding maximum value of the $S_{2}{ }^{2 c}$-type velocity $\frac{8}{3} \cdot V_{2,2 c}$ becomes $\frac{8}{3} V_{2,2 c}=0.01 \frac{\mathrm{cm}}{\mathrm{sec}}$.

[2] The energy dissipation $W_{1}$ by viscosity (the kinetic energy transformed into heat by friction) per unit time is

$$
W_{1}=\mu \int(\operatorname{curl} V)^{2} d v,
$$

where $\mu$ is the coefficient of viscosity. The integration in (5.1) extends through the earth's core. Since we are studying the case in which $X \rightarrow \infty$, it is sufficient for our purpose to take the $T_{1}{ }^{0}$-type velocity only in the $V$ in (5.1). The value of $W_{1}$ thus calculated is

$$
W_{1}=\frac{8 \pi a \mu}{15}\left(V_{1,0}\right)^{2}=5.5 \times 10^{7} \mu \mathrm{erg} \text { sec. }
$$

in which $a$ and $V_{1,0}$ are taken to be the same as in [1].

[3] The energy $W_{2}$ dissipated as the JouLE's heat per unit time is

$$
W_{2}=\frac{1}{\kappa} \int I^{2} d v=\frac{1}{(4 \pi)^{2} \kappa} \int(\operatorname{curl} H)^{2} d v .
$$

Taking only the $T_{2}{ }^{0}$-type magnetic field into consideration, we have

$$
W_{2}=0.075 \kappa a\left(a V_{1,0}\right)^{2}=8.8 \times 10^{17} \frac{\mathrm{erg} .}{\mathrm{sec} .}
$$

The energy dissipation takes its maximum value at $\xi=1$, i.e., near the surface of the earth's core. Since the mass of thc core is $1.9 \times 10^{27} \mathrm{gr}$., the corresponding JouLe's dissipation in each gram of the core is $1.1 \times 10^{-17}$ cal. sec.

[4] The ratio of the energy dissipation by the mechanical friction to that by the Joune's heat is given by (5.2) and (5.4). This will become equal to $I$ for the viscosity $\mu$ of the order of $10^{10} \mathrm{c.g} . \mathrm{s}$. . If the viscosity of the earth's core is smaller than this critical value, the energy dissipation by viscosity becomes negligible compared with that by the Joune's heat. 
[5] The energy dissipation $W_{2}$ of the field is compensated by the work done on the field by the fluid motion. This will be understood as follows. The pondermotive force $F$ of the field upon the current is given by the well-known Fueming's law

$$
F=I \times H=\frac{1}{4 \pi} \operatorname{curl} H \times H .
$$

Taking the negative of the work done on the fluid by this force per unit time, we have

$$
W_{3}=-\int V \cdot F d v=\frac{1}{4 \pi} \int V \cdot(H \times \operatorname{curl} H) d v,
$$

the integral extending through the earth's core. This is nothing but the work done on the field by the fluid motion. Using the results obtained in the last section, we have

$$
W_{3}=8.8 \times 10^{17} \frac{\mathrm{erg}}{\mathrm{sec}}=W_{2} .
$$

Thus it is shown that the energy flow in the course of hydromagnetic interchange is from the fluid motion to the field. The work $W_{3}$ takes its maximum value at about $\xi=\frac{2}{3}$. In short, the field takes its energy in the deeper part of the earth's core and consumes it near the surface of the core.

[6] If the fluid motion and the field are to be stationary, there must be a source of energy which compensates the energy dissipation by the mechanical friction and the JoulE's heat. The energy transformation in our self-exciting dynamo may thus be shown schematically as in Fig. 5. Taking the results in [3] and [4] into consideration, and taking $\mu=10^{10} \mathrm{c} . \mathrm{g} . \mathrm{s}$. tentatively, the required energy is estimated to be of the order of $2 \times 10^{-17}$

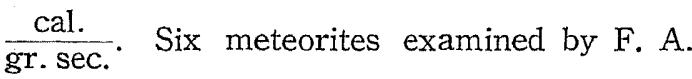
PANeth (1942) were found to contain radioactive substances, and to generate $4 \times 10^{-16}$ $\frac{\text { cal. }}{\text { gr. sec. }}$ This is about twenty times as large an amount as that required above.

[7] The current density $I$ at the surface of the earth's core is calculated by the rela-

\section{ENERGY SOORCE}

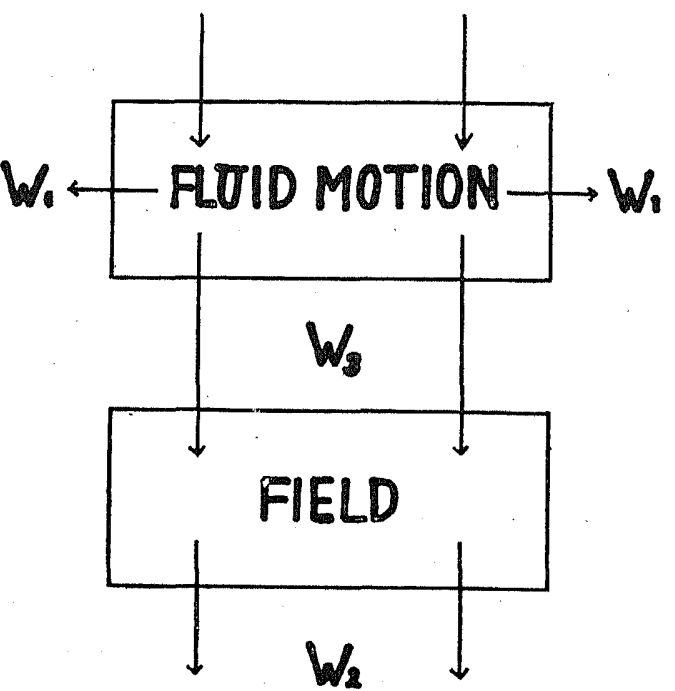

Fig. 5. Schematic representation of the energy transformation in the present model of the selfexciting dynamo.

tion $I=\frac{1}{4 \pi}$ curl $H$. Taking only the $T_{2}{ }^{\prime \prime}$-type magnetic field into account, we have the maximum value of $I$ of the order of $2.7 \times 10^{-7}$ e.m.u. Two-dimensional current sheet of this current density and the skin depth of about $10 \mathrm{~km}$. will give values for the current densities near the surface of the core, which are appropriate to explain the secular variations in the earth's magnetic field.

$\S 6$. In the last section, we have examined the case when $X=1000$. No absurdity was met with in the course of this examination. In order to complete the study of the selfexciting dynamo, however, there remains a difficult problem to solve. That is the problem on the fluid motion. Is it possible that such a fluid motion takes place in the earth's core? Is the fluid motion appropriate to maintain the self-exciting dynamo supposed in the present paper?

Putting aside these problems for a while, we shall now show that the self-exciting dynamo is possible by the fluid motions of considerable varieties. In (2.1), we put 


$$
\begin{aligned}
& V_{2}{ }^{2 c}(r)=\frac{V_{2,2 c}}{a}\left[1-\left(\frac{r}{a}\right)\right]^{2}, \\
& V_{1}{ }^{0}(r)=\frac{V_{1,0}}{a}\left[1-\left(\frac{r}{a}\right)\right],
\end{aligned}
$$

tentatively, for the radial parts of the velocity fields. There is, however, no reason why the radial functions should be of these types. In order to make the matter clearer, studies will be made whether or not the self-exciting dynamo is possible by the fluid motions of the following types

$$
\begin{aligned}
& V_{2}{ }^{2 c}(r)=\frac{V_{2,2 c}}{a} V_{2}{ }^{2 c}(\xi), \quad V_{2}{ }^{2 c}(\xi)=\xi^{\sigma}(1-\xi)^{2}, \\
& V_{1}^{0}(r)=\frac{V_{1,0}}{a} V_{1}^{0}(\xi), \quad V_{1}^{0}(\xi)=\xi^{\tau}(1-\xi), \\
& \xi=\frac{r}{a},
\end{aligned}
$$

where $\sigma$ and $\tau$ are certain integers. By the same reason as is stated in $\S 2$, we shall confine ourselves to the study of the case in which $\frac{V_{1,0}}{V_{2,26}} \rightarrow \infty$. Thus, as in $\S 5$ in Paper $I$, the problem is reduced to solve

$$
\begin{aligned}
& T_{2}{ }^{2 s}(\xi)=\frac{3 y}{7 \xi^{3} V_{1}^{0}(\xi)} \\
& \times\left[\frac{d}{d \xi}\left(\xi^{4} V_{2}{ }^{2 c} \bar{T}_{2}{ }^{0}\right)+\frac{d}{d \xi}\left(\xi^{3} V_{2}{ }^{2 c}\right) \xi \bar{T}_{2}{ }^{0}\right], \\
& \frac{d^{2} S_{1}{ }^{0}}{d \xi^{2}}+\frac{4 d S_{1}{ }^{0}}{\xi}=\frac{9 \times 24}{5} y \xi^{2} V_{2}^{2 c}(\xi) T_{2}{ }^{2 s}, \\
& y=4 \pi \kappa a V_{2,2 c}, \\
& \frac{d^{2} \bar{T}_{2}^{0}}{d \xi^{2}}+\frac{6 d \bar{T}_{2}^{0}}{\xi d \xi}=\frac{2 d V_{1}^{0}}{d \xi} S_{1}^{0},
\end{aligned}
$$

under the boundary conditions

$$
\begin{aligned}
& \bar{T}_{2}{ }^{0}(\xi)=0, \\
& \frac{d S_{1}{ }^{0}}{d \xi}+\frac{3}{\xi} S_{1}{ }^{0}=0,
\end{aligned}
$$

at $\xi=1$.

It is, however, almost hopeless to solve the eigen-value problem for $y$ by the trial and error method. We shall solve the problem by an approximate way which is similar to. that adopted in $\$ 4$ of Paper I. At first, taking (6.6) into consideration, we shall put

$$
\begin{aligned}
& \bar{T}_{2}^{0}(\xi)=A_{0}(1-\xi)+A_{1} \xi(1-\xi)+\ldots \\
& \quad+A_{n} \xi^{n}(1-\xi)+\ldots,
\end{aligned}
$$

for $\bar{T}_{2}{ }^{0}(\xi)$. $A_{n}$ 's $(n=0,1, \ldots)$ in $(6.8)$ are certain constants to be determined. The radial function

$$
\bar{T}_{2}{ }^{0}(\xi, n)=\xi^{n}(1-\xi),
$$

under $A_{n}$ will be called the $n$-th coordinate function. Inserting (6.9) in (6.3) and (6.4) and taking (6.7) into consideration, we get

$$
S_{1}{ }^{0}(\xi)=\frac{24 \times 27}{35}-y^{2} \sum A_{n} S_{1}^{0}(\xi, n)
$$

in which $S_{1}{ }^{0}(\xi, n)$ is

$$
\begin{aligned}
& S_{1}{ }^{0}(\xi, n)=-\frac{1}{3}\left(\frac{n^{\prime}+7}{N+4}-\frac{4 n^{\prime}+33}{N+5}+\frac{6 n^{\prime}+57}{N+6}\right. \\
& \left.-\frac{4 n^{\prime}+43}{N+7}+\frac{n^{\prime}+12}{N+8}\right)+\frac{n^{\prime}+7}{(N+4)(N+7)^{-1.1}} \xi^{N+1} \\
& -\frac{4 n^{\prime}+33}{(N+5)(N+8)} \xi^{N+5}+\frac{6 n^{\prime}+57}{(N+6)(N+9)^{\xi}} \xi^{N+0} \\
& -\frac{4 n^{\prime}+43}{(N+7)(N+10)^{\xi+7}} \xi^{N+7} \\
& +\frac{n^{\prime}+12}{(N+8)(N+11)^{\xi^{N+8}}}
\end{aligned}
$$

In (6.11), $n^{\prime}$ and $N$ are

$$
n^{\prime}=n+2 \sigma, \quad N=n^{\prime}-\tau=n+2 \sigma-\tau,
$$

respectively. The constants $A_{n}$ 's will be determined by the following orthogonality conditions (see $\$ 3$ and $\S 4$ of Paper I)

$$
\begin{aligned}
& \int_{0}^{1} \xi^{0} \bar{T}_{2}^{0}(\xi, n)\left[\frac{d^{2} \bar{T}_{2}^{0}}{d \xi^{2}}+\frac{6 \cdot d \bar{T}_{2}^{0}}{\xi} \frac{2 d V_{1}^{0}}{d \xi}-3 \xi \frac{V_{1}^{0}}{d \xi} S_{1}{ }^{0}\right] d \xi \\
& \quad=0, \quad(n=0,1, \ldots .),
\end{aligned}
$$

in which $\bar{T}_{2}^{0}$ and $S_{1}{ }^{0}$ are to be taken as those in (6.8) and (6.10). If we take (6.8) into consideration, we see at once that (6.13) is nothing but the equation from which (6.5) itself is obtained. Executing the integral in (6.13), we get the equations of the same types as those in Table I of Paper I. Equating the determinant formed by the coefficients of $A_{n}$ 's to zero, we have the equation by which the eigen-value $y=4 \pi \kappa a V_{2, y c}$ is determined. By the present method, better approximation for $y$ is obtained with less numerical computations than by that in Paper I. In fact, putting $\sigma=\tau=0$ in (6.2) and using the method in the present paper, we have the value of $Y=\frac{8}{3} y$ as is shown in Table VI. In Table VI, the second approximation, for example, means the value of $Y$ which is obtained by 
using two coordinates functions and solving the matrix equation of rank 2. From this table, we see that our approximate values for $Y$ agree with exact eigen-value satisfactorily. Looking at the result from a different angle, we may say that the result in $\$ 2$ and $\$ 3$ of the present paper is checked in an independent way.

Table VI. The eigen-value for the case $\sigma=\tau=0$ obtained by the new approximate method.

\begin{tabular}{c|c}
\hline approximation & $Y$ \\
\hline 1 & 111.7 \\
2 & $1 \% 9.3$ \\
3 & 130.2 \\
exact & 130.6 \\
\hline
\end{tabular}

\$7. Returning to our main subject, we shall now sudy the case in which $\sigma=\tau \Leftrightarrow 0)$. For the larger value of $\sigma=\tau$, the depth of the fluid motion becomes the shallower. In fact, the value of $\xi$ for which the fluid velocity becomes maximum is given by

$$
\xi=\left(\xi_{m}, \text { say }\right)=\frac{\sigma+1}{\sigma+3} .
$$

The maximum value of the radial velocity of $S_{2}{ }^{2 c}$-type velocity field is given by

$$
\text { Max. vel. }=18 \xi_{m}^{\sigma+1}\left(1-\xi_{m}\right)^{2} V_{2,2 c} \text {, }
$$

where $V_{2,20}$ is the constant in (6.2). Defining $Y$ by the following equation

$$
Y=4 \pi \kappa a \cdot(\text { Max. vel. }),
$$

the value of $Y$ is calculated for several $\sigma=\tau$ up to the second approximation. The first approximation for $Y$ differs but little from the second approximation which is shown in Table VII. From Table VII, we see that the self-exciting dynamo is possible for all $\sigma=\tau$ studied, and the values of $Y$ obtained do not differ substantially from that for $\sigma=\tau=0$ in Paper I. In addition to the results in Table VII, the values of $Y$ are calculated for several values of $\sigma$ and $\tau(\neq \sigma)$. Only the first approximation for $Y$ is calculated, and the results of calculations are shown in Table VIII.
Table VII

Table VIII

The eigen-values for various types of fluid motions.

\begin{tabular}{c|r}
\hline$\sigma=\tau$ & \multicolumn{1}{|c}{$Y$} \\
\hline 0 & 129.3 \\
2 & 98.2 \\
4 & 44.5 \\
8 & 28.9 \\
16 & 25.6 \\
\hline
\end{tabular}

\begin{tabular}{c|c|c}
\hline$\sigma$ & $\tau$ & $Y$ \\
\hline 2 & 0 & 56.2 \\
4 & 0 & 42.2 \\
8 & 0 & 32.2 \\
0 & 2 & 154.0 \\
0 & 8 & 90.8 \\
\hline
\end{tabular}

In view of the results obtained in this section, we may say that the self-exciting dynamo is possible by the fluid motions of considerable varieties. The only condition required for the existence of the self-exciting dynamo is that the radial velocity is of the order of magnitude for which $Y$ in (7.3) becomes

$$
Y \approx 10^{2} .
$$

Putting $a=3.5 \times 10^{8} \mathrm{~cm}$. and $\kappa=3 \times 10^{-6}$ e.m.u., the corresponding maximum radial velocity is calculated to be

$$
\text { Max. vel. }=0.01 \mathrm{~cm} \text { sec. }
$$

Is it possible that the fluid motion, of this order of magnitude, takes place in the earth's core? Under what condition is the fluid motion possible? It, thus, becomes desirable to study these problems rather in detail. This will be done in a forthcoming paper.

\section{References}

BULLARD, E.C. :

1948 "The secular change in the earth's magnetic field". Mon. Not. Roy. Astron. Soc., Geophys. Suppl., 5, 248.

1949 "The magnetic field within the earth". Proe. Roy. Soc., 197, 433 .

"Electromagnetic induction in a rotating sphere". Proc. Roy. Soc., 199, 413.

Elsasser, W.M. :

1946 " Induction effects in terrestrial magnetism (Part 1)". Phys. Rev., 69, 10ti.

"Induction elfects in terrestrial magnetism (Part 2)". Phys. Rev., 70, 202.

1947 " Induction effects in terrestrial magnetism (Part 3)". Phys. Rev,, 72, 821.

PANeTh, F.A. :

1942 Nature, 149, 235.

TAKEUCh, H. and ShrmazU, Y.:

1952 "On a self-exciting process in magnetohydrodynamies". Journ. Phys. Earth, 1, 1 . 